\title{
Intracellular localization of mesothelin predicts patient prognosis of extrahepatic bile duct cancer
}

\author{
FUTOSHI KAWAMATA $^{1}$, HIROFUMI KAMACHI ${ }^{1}$, TAKAHIRO EINAMA ${ }^{1}$, SHIGENORI HOMMA ${ }^{1}$, \\ MUNENORI TAHARA ${ }^{1}$, MASAYA MIYAZAKI ${ }^{3}$, SHINYA TANAKA ${ }^{3}$, TOSHIYA KAMIYAMA ${ }^{1}$, \\ HIROSHI NISHIHARA ${ }^{2}$, AKINOBU TAKETOMI $^{1}$ and SATORU TODO ${ }^{1}$ \\ Departments of ${ }^{1}$ General Surgery, ${ }^{2}$ Translational Pathology and ${ }^{3}$ Cancer Pathology, \\ Hokkaido University School of Medicine, Sapporo 060-8638, Japan
}

Received July 30, 2012; Accepted September 14, 2012

DOI: 10.3892/ijo.2012.1662

\begin{abstract}
Mesothelin is expressed in various types of malignant tumors, and we recently reported that the expression of mesothelin was related to unfavorable patient outcome in pancreatic ductal adenocarcinoma and gastric adenocarcinoma. In this study, we examined the clinicopathological significance of mesothelin expression in extrahepatic bile duct cancer (EHBDCA), especially in terms of its association with the staining pattern. Tissue samples from 61 EHBDCA (16 hilar cholangiocarcinoma, 17 upper bile duct adenocarcinoma, 20 middle bile duct adenocarcinoma and 8 distal bile duct adenocarcinoma) were immunohistochemically examined. The expression levels of mesothelin in tumor cells was classified into the localization of mesothelin in luminal membrane and/or cytoplasm, in addition to high and low according to the staining intensity and proportion as a conventional analysis. 'High-level expression' of mesothelin (47.5\%) was statistically correlated with liver metastasis $(\mathrm{P}=0.013)$ and poorer patient outcome $(\mathrm{P}=0.022)$, while 'luminal membrane positive' of mesothelin $(52.5 \%)$ was more significantly correlated with liver metastasis $(\mathrm{P}=0.006)$, peritoneal metastasis $(\mathrm{P}=0.024)$ and unfavorable patient outcome $(\mathrm{P}=0.017)$. Moreover, we found that 'cytoplasmic expression' isolated from 'luminal membrane negative' of mesothelin represented the best patient prognosis throughout this study. We describe the expression pattern level of mesothelin, i.e., in luminal membrane or cytoplasm both high and low level, evidently indicate the patient prognosis of EHBDCA, suggesting the pivotal role of mesothelin in cancer promotion depending on its intracellular localization.
\end{abstract}

Correspondence to: Dr Hiroshi Nishihara, Department of Translational Pathology, Hokkaido University School of Medicine, Kita-Ku, Kita 14, Nishi 7, Sapporo 060-8638, Japan

E-mail: hnishihara@s5.dion.ne.jp

Key words: mesothelin, intracellular localization, luminal membrane expression

\section{Introduction}

Extrahepatic bile duct cancer (EHBDCA), consisting of hilar cholangiocarcinoma and distal bile duct adenocarcinoma (excluding gallbladder cancer), is a rare disease in the United States with an incidence of 1-2/100,000/year (1). It occurs with great frequency in Asian countries, and is one of the common causes of cancer death in Japan, with near to 17,000 deaths annually (2). The 5-year survival rate of EHBDCA, even after the surgical resection is poor, ranging from 20 to $45 \%$ (3-5). The incidence of EHBDCA is increasing throughout the world with a high fatality rate; therefore, new prognostic markers and treatment for EHBDCA patients are urgently needed.

Mesothelin is expressed on normal mesothelial cells lining the pleura, pericardium and peritoneum $(6,7)$. In addition, the overexpression of mesothelin has been found in several cancer types, including malignant mesothelioma, ovarian cancer and pancreatic cancer $(8-11,12)$. The full length of human mesothelin gene codes the primary product, which is a $71-\mathrm{kDa}$ precursor protein. This protein can be physiologically cleaved by certain furin-like proteases into a $40-\mathrm{kDa} C$-terminal fragment that remains membrane-bound and a $31-\mathrm{kDa} N$-terminal fragment, which is secreted into the blood (6). The $C$-terminal $40-\mathrm{kDa}$ fragment is named mesothelin and is attached to the cell membrane through a glycosyl-phosphatidylinositol (GPI) anchor (13). The biological functions of mesothelin are not clearly understood, although recent studies have suggested that enforced expression of mesothelin increases cell proliferation and migration (14). In ovarian cancers, higher mesothelin expression was found to be associated with chemoresistance and shorter patient survival (15). In pancreatic cancer, mesothelin expression was immunohistochemically observed in all cases, while its absence was noted in non-cancerous pancreatic ductal epithelium, with or without pancreatitis $(8,12,16,17)$. We recently found that the expression of mesothelin was related to an unfavorable patient outcome in pancreatic ductal adenocarcinoma (12), while the opposite result was reported in gastric cancer, in which the mesothelin expression was correlated with prolonged patients' survival (18). However, our consecutive investigation for mesothelin expression patterns in gastric cancer recently discovered that luminal membrane expression, not cytoplasmic expression 
of mesothelin is a prominent negative prognostic factor for gastric cancer (19), suggesting the significance of expression pattern of mesothelin in clinicopathological analysis of cancer. In EHBDCA, Zhao et al, who first studied mesothelin expression in dysplasia and carcinoma of external bile duct, reported that mesothelin was expressed in 5 of 10 adenocarcinomas $(50 \%)$ in cell membranes and cytoplasm (20); however, the detailed clinicopathological analysis of mesothelin expression in EHBDCA, especially with large number of the cases, has not yet been performed.

In this study, we investigated the mesothelin expression in 61 EHBDCA cases by immunohistochemistry, and its clinicopathological significance associated with patients' outcome was analyzed. Moreover, we focused on the intracellular localization of mesothelin, i.e., in luminal membrane and/or cytoplasm, and its clinicopathological significance associated with the patients' outcome.

\section{Materials and methods}

Patients' demography and tumor specimens. This study was performed with the approval of the Internal Review Board on Ethical Issues of Hokkaido University Hospital, Sapporo, Japan. The samples and the patient information were obtained under a blanket written informed consent. The subjects of this study were 61 patients who underwent radical surgery for bile duct adenocarcinoma between the years 2000 and 2008 at Hokkaido University Hospital by the Department of General Surgery, Hokkaido University, Graduate School of Medicine, Sapporo, Japan. The clinicopathological characteristics of these cases are summarized in Table I.

Mean age of patients was 67.5 years $[ \pm 9.0$ standard deviation (SD)]; 47 patients $(77.0 \%)$ were male and 14 patients $(23.0 \%)$ were female. The predominant sites of the cancer were the hilar bile duct in 16 cases $(26.2 \%)$, upper bile duct in 17 cases $(27.9 \%)$, middle bile duct in 20 cases $(32.8 \%)$ and distal bile duct in 8 cases (13.1\%). The surgical procedures consisted of the standard pancreatoduodenectomy in $21(34.4 \%)$ cases, the pylorus-preserving pancreatoduodenecomy in 5 cases $(8.2 \%)$, the extended right or left hemihepatectomy with extrahepatic bile duct resection in 28 cases $(45.9 \%)$, and the extrahepatic bile duct resection in 7 cases $(11.5 \%)$. Intraoperative diagnosis of the ductal resection margins was performed using frozen sections. When a positive margin was found, additional resection of marginal bile duct was performed to the maximum extent possible. R0 curative resection was achieved in 39 cases $(63.9 \%)$, and R1 resection was achieved in 22 cases (36.1\%). T-factor, $\mathrm{N}$-factor, $\mathrm{M}$-factor and clinical stage were assigned according to the TNM classification of the Union Internationale Contre le Cancer (UICC) (21). The median survival time of patients was 29.8 months $( \pm 3.5 \mathrm{SD})$.

Formalin-fixed paraffin-embedded tissue blocks were prepared from surgical specimens and sections were sliced and stained with hematoxylin and eosin (H\&E) for routine histopathological examination. All specimens were diagnosed as EHBDCA.

Immunohistochemical evaluation. Immunohistochemical staining against mesothelin was performed as described
Table I. Clinicopathological characteristics of 61 patients with EHBDCA in this study.

\begin{tabular}{|c|c|}
\hline Parameter & No. of cases \\
\hline \multicolumn{2}{|l|}{ Age (years) } \\
\hline$<60$ & 11 \\
\hline$\geq 60$ & 50 \\
\hline Mean \pm SD & $67.5 \pm 9.0$ \\
\hline \multicolumn{2}{|l|}{ Gender } \\
\hline Male & 47 \\
\hline Female & 14 \\
\hline \multicolumn{2}{|l|}{ Location } \\
\hline Hilar & 16 \\
\hline Upper & 17 \\
\hline Middle & 20 \\
\hline Distal & 8 \\
\hline \multicolumn{2}{|l|}{ Surgical procedure } \\
\hline Pancreatoduodenectomy & 21 \\
\hline Pylorus-preserving pancreatoduodenectomy & 5 \\
\hline $\begin{array}{l}\text { Extended right or left hemihepatectomy } \\
\text { with bile duct resection }\end{array}$ & 28 \\
\hline Extrahepatic bile duct resection & 7 \\
\hline \multicolumn{2}{|l|}{ Resection status } \\
\hline R0 & 39 \\
\hline $\mathrm{R} 1$ & 22 \\
\hline \multicolumn{2}{|l|}{ T-factor } \\
\hline $\mathrm{T} 1$ & 5 \\
\hline $\mathrm{T} 2$ & 27 \\
\hline $\mathrm{T} 3$ & 19 \\
\hline $\mathrm{T} 4$ & 10 \\
\hline \multicolumn{2}{|l|}{$\mathrm{N}$-factor } \\
\hline NO & 25 \\
\hline N1 & 36 \\
\hline \multicolumn{2}{|l|}{ M-factor } \\
\hline M0 & 58 \\
\hline M1 & 3 \\
\hline \multicolumn{2}{|l|}{ Stage } \\
\hline IA & 4 \\
\hline IB & 14 \\
\hline IIA & 4 \\
\hline IIB & 28 \\
\hline III & 8 \\
\hline IV & 3 \\
\hline Median survival (months) & $29.8 \pm 3.5$ \\
\hline
\end{tabular}

SD, standard deviation.

previously (12). In brief, the tissue sections were incubated with a mouse monoclonal antibody against mesothelin (clone 5B2 diluted 1:50; Novocastra, Newcastle Upon Tyne, $\mathrm{UK})$ at a 1:50 dilution, and reacted with a dextran polymer reagent combined with secondary antibodies and peroxidase (Envision/HRP; Dako). All assessments were made 

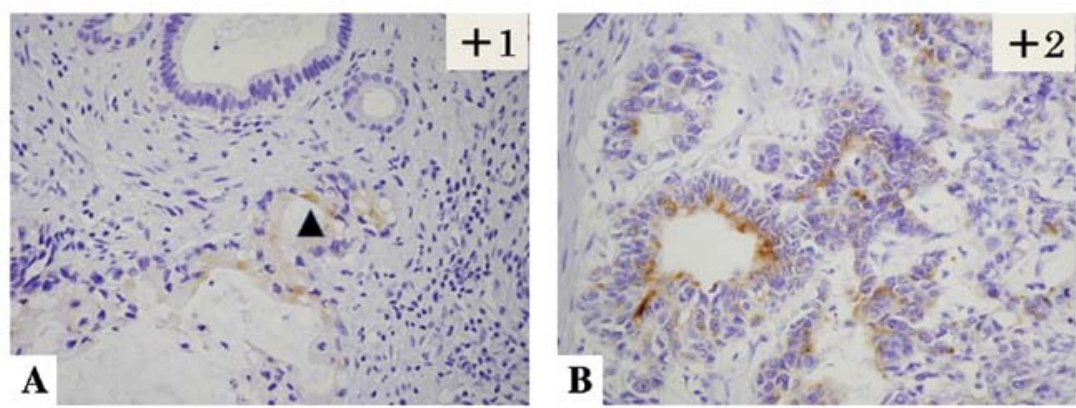

Figure 1. Representative cases of 'low-level expression' (A) and 'high-level expression' (B) of mesothelin in EHBDCA specimens by immunoistochemistly (A) Partial luminal membrane staining (arrowhead; intensity, +1 ) and the weak cytoplasmic staining were observed in $<50 \%$ area (proportion, +2 ). (B) Entire circumference of the luminal membrane was strongly positive in $>50 \%$ tumor cells (intensity, +2 ; proportion, +3 ). (Magnification, $x 200$ ).
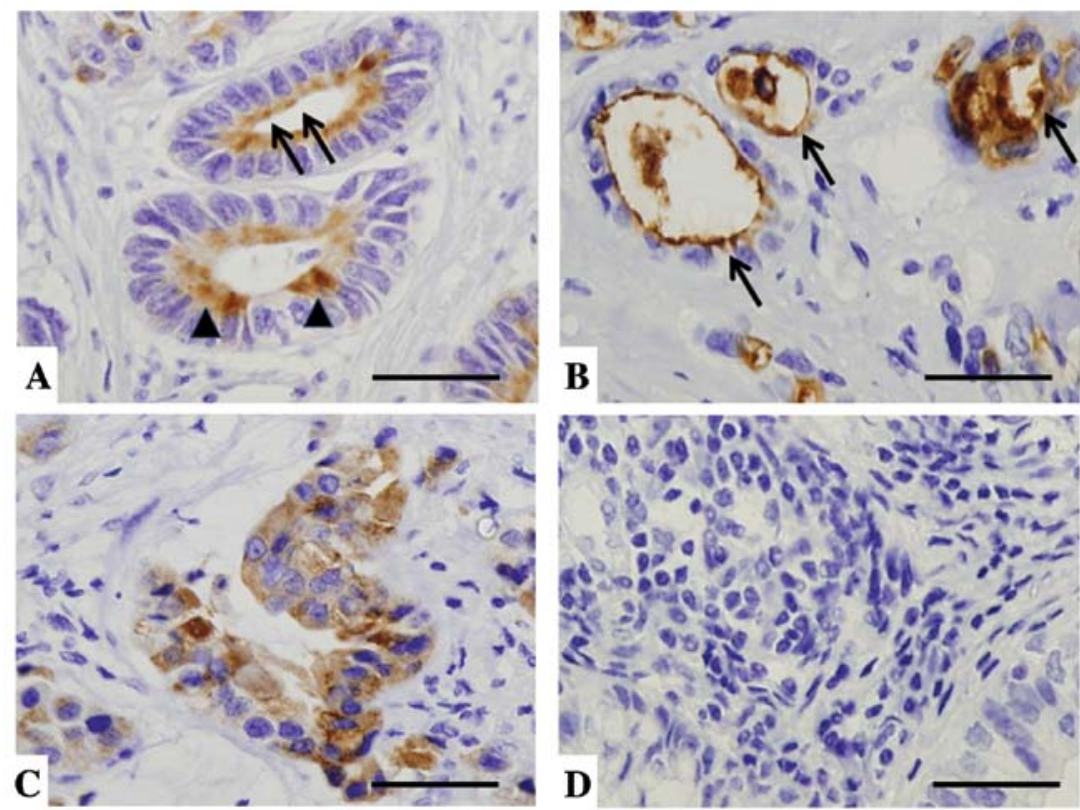

Figure 2. Representative cases of 'luminal membrane positive' (A, B) and 'luminal membrane negative' (C, D) of mesothelin in EHBDCA specimens by immunohistochemistry. (A) Granular cytoplasmic staining was observed (arrowheads; intensity, +2 ) and luminal membrane was also stained partially (arrows). (B) Entire circumference of the luminal membrane was explicitly stained (arrows). (C) Granular cytoplasmic, but no membranous staining in cancer cells was observed. (D) No expression of mesothelin was found in tumor cells, also designated 'mesothelin negative'. (Magnification, $\mathrm{x} 400$; scale bars, $50 \mu \mathrm{m}$ ).

on the tumor region of the specimen (x400). Each slide was evaluated independently by three pathologists (F. Kawamata, M. Miyazaki and H. Nishihara) who did not know the clinical outcomes. Immunostaining for mesothelin was evaluated for both the proportion and staining intensity of tumor cells in each case. The proportion of mesothelin expression was assessed according to the percentage of mesothelin-positive cells as follows: $0,0 \% ;+1,1<10 \% ;+2,10-50 \%$; and $+3,>50 \%$. The staining intensity of mesothelin was evaluated as weak $(+1)$ and moderate to strong (+2) (Table II). The final evaluation of mesothelin expression was assessed using the following scoring system: 'high-level expression' of mesothelin was defined as $\geq+3$ of the proportion score and/or +2 of the intensity score, while a 'low-level expression' of mesothelin was given when the total score was $\leq+3$ except in cases when the proportion score was +1 and the intensity score was +2 (Fig. 1).

Furthermore, among the 61 cases of EHBDCA, the staining localization of mesothelin was evaluated in luminal membrane
Table II. Immunohistochemical findings of mesothelin expression.

\begin{tabular}{|c|c|c|c|c|}
\hline \multirow{3}{*}{$\begin{array}{l}\text { Staining intensity } \\
\text { on tumor cells }\end{array}$} & \multicolumn{4}{|c|}{ No. of cases $(\%)$} \\
\hline & \multicolumn{4}{|c|}{ Percentage of mesothelin-positive cells } \\
\hline & 0 & $1-10 \%$ & $10-50 \%$ & $>50 \%$ \\
\hline Score 0 & $17(27.9)$ & $0 \quad(0.0)$ & $0 \quad(0.0)$ & $0 \quad(0.0)$ \\
\hline Score 1 & $0 \quad(0.0)$ & $13(21.3)$ & $2(3.3)$ & 1 (1.6) \\
\hline Score 2 & $0 \quad(0.0)$ & $6(9.8)$ & $12(19.7)$ & $10(16.4)$ \\
\hline
\end{tabular}

or cytoplasm. Cases in which the luminal membrane was stained even partially or faintly (Fig. 2A), or the entire circumference of the luminal membrane was explicitly stained 
Table III. Correlation between mesothelin expression levels and clinicopathological features.

\begin{tabular}{|c|c|c|c|c|c|c|c|}
\hline \multirow[b]{2}{*}{ Parameter } & \multirow[b]{2}{*}{ Total } & \multicolumn{3}{|c|}{ Mesothelin } & \multicolumn{3}{|c|}{ Luminal membrane expression } \\
\hline & & $\begin{array}{l}\text { High-level } \\
\quad(\mathrm{n}=29)\end{array}$ & $\begin{array}{l}\text { Low-level } \\
(\mathrm{n}=32)\end{array}$ & P-value & $\begin{array}{c}\text { Positive } \\
(n=32)\end{array}$ & $\begin{array}{l}\text { Negative } \\
(n=29)\end{array}$ & P-value \\
\hline \multicolumn{8}{|c|}{ Histopahological grade } \\
\hline 1 or 2 & 54 & 26 & 28 & 1.000 & 28 & 26 & 1.000 \\
\hline 3 & 7 & 3 & 4 & & 4 & 3 & \\
\hline \multicolumn{8}{|l|}{ pT-factor } \\
\hline pT1-2 & 32 & 13 & 19 & 0.310 & 19 & 13 & 0.310 \\
\hline pT3-4 & 29 & 16 & 13 & & 13 & 16 & \\
\hline \multicolumn{8}{|l|}{ pN-factor } \\
\hline Negative & 25 & 11 & 14 & 0.795 & 16 & 9 & 0.193 \\
\hline Positive & 36 & 18 & 18 & & 16 & 20 & \\
\hline \multicolumn{8}{|l|}{ pStage } \\
\hline I-IIB & 50 & 24 & 26 & 1.000 & 26 & 24 & 1.000 \\
\hline III-IV & 11 & 5 & 6 & & 6 & 5 & \\
\hline \multicolumn{8}{|c|}{ Lymphatic permeation } \\
\hline Negative & 23 & 10 & 13 & 0.792 & 12 & 11 & 1.000 \\
\hline Positive & 38 & 19 & 19 & & 20 & 18 & \\
\hline \multicolumn{8}{|c|}{ Blood vessel permeation } \\
\hline Negative & 26 & 11 & 15 & 0.606 & 11 & 15 & 0.200 \\
\hline Positive & 35 & 18 & 17 & & 21 & 14 & \\
\hline \multicolumn{8}{|c|}{ Perineural invasion } \\
\hline Negative & 9 & 3 & 6 & 0.478 & 3 & 6 & 0.287 \\
\hline Positive & 52 & 26 & 26 & & 29 & 23 & \\
\hline \multicolumn{8}{|c|}{ Resection margin } \\
\hline $\mathrm{pR} 0$ & 39 & 20 & 19 & 0.594 & 24 & 15 & 0.069 \\
\hline $\mathrm{pR} 1$ & 22 & 9 & 13 & & 8 & 14 & \\
\hline \multicolumn{8}{|l|}{ Recurrence } \\
\hline No & 18 & 6 & 12 & 0.172 & 6 & 12 & 0.090 \\
\hline Yes & 43 & 23 & 20 & & 26 & 17 & \\
\hline \multicolumn{8}{|c|}{ Liver metastasis } \\
\hline No & 47 & 18 & 29 & 0.013 & 20 & 27 & 0.006 \\
\hline Yes & 14 & 11 & 3 & & 12 & 2 & \\
\hline \multicolumn{8}{|c|}{ Local recurrence } \\
\hline No & 46 & 22 & 24 & 1.000 & 25 & 21 & 0.767 \\
\hline Yes & 15 & 7 & 8 & & 7 & 8 & \\
\hline \multicolumn{8}{|c|}{ Peritoneal metastasis } \\
\hline No & 49 & 20 & 29 & 0.052 & 22 & 27 & 0.024 \\
\hline Yes & 12 & 9 & 3 & & 10 & 2 & \\
\hline
\end{tabular}

(Fig. 2B) were judged as 'luminal membrane positive'. In cases with no membrane staining (Fig. 2D) and those in which only cytoplasmic staining (Fig. 2C) was observed in any intensity level, the term 'luminal membrane negative' was given.

Statistical analysis. We used the $\chi^{2}$ test or Fisher's exact test to determine the correlation between mesothelin and clinicopathologic data. Survival curves for patients were drawn by the Kaplan-Meier method. Differences in survival curves were analyzed by the log-rank test. Prognostic implications of mesothelin expression and clinicopathologic parameters were analyzed by Cox univariate and multivariate proportional hazards models. All differences were considered significant at a P-value of $<0.05$. All statistical analyses were performed using the Ekuseru-Toukei 2010 software for Windows (Social Survey Research Information Co., Ltd., Tokyo, Japan).

\section{Results}

High-level expression of mesothelin was correlated with liver metastasis and poor patient outcome. The overexpression of mesothelin has been found in several cancer types, including 


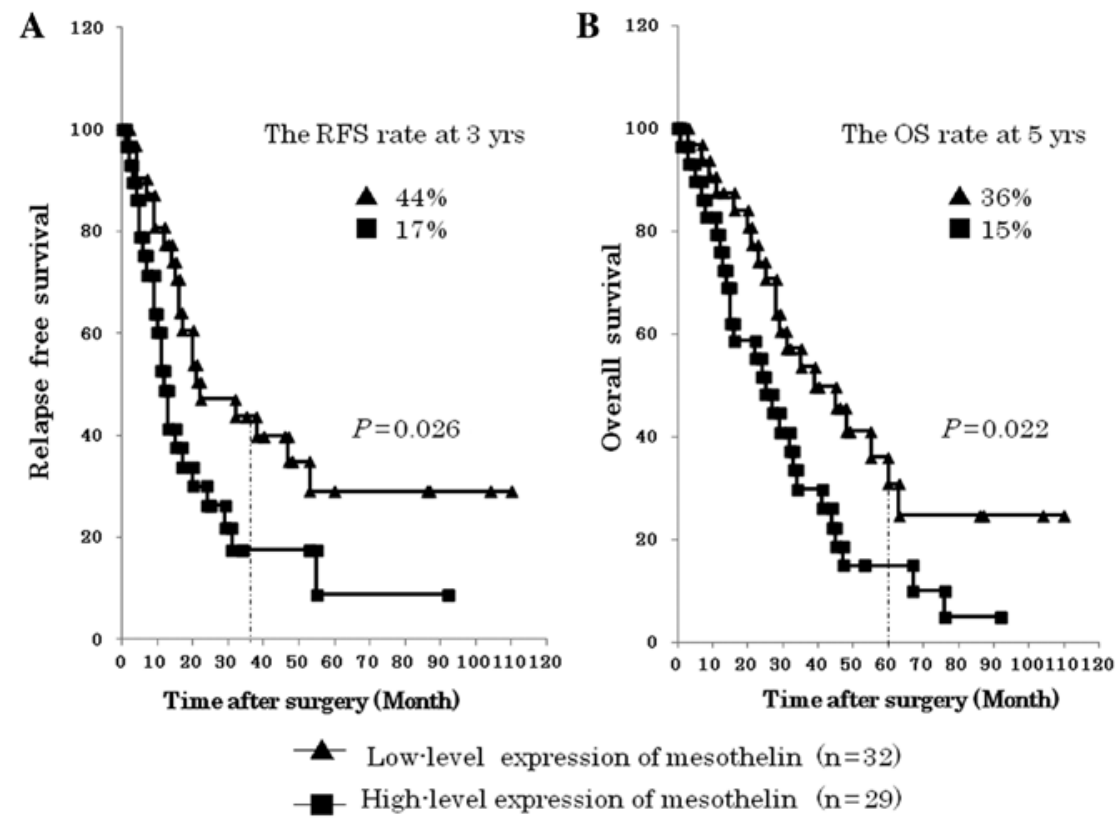

Figure 3. Relapse-free survival (RFS) and overall survival (OS) curves of EHBDCA patients according to the expression levels of mesothelin. The group of 'high-level expression' of mesothelin represented a statistically significantly unfavorable outcome compared to the group of 'low-level expression' ( $\mathrm{P}=0.026$ and 0.022 , respectively).

malignant mesothelioma, ovarian cancer, and pancreatic cancer $(8-11,12)$; thus, we first evaluated the comprehensive expression of mesothelin in EHBDCA. As described in Materials and methods, 'high-level expression' and 'low-level expression' of mesothelin was attributed to all 61 cases of EHBDCA (Fig. 1). As summarized in Table II, 'high-level expression' was detected in 29 cases $(47.5 \%)$, whereas 'low-level expression' was detected in 32 cases $(52.5 \%)$. The statistical analysis for the clinicopathological parameters such as histological grade, T-factor and metastasis revealed that 'high-level expression' of mesothelin was significantly correlated with liver metastasis $(\mathrm{P}=0.013$, Table III). Furthermore, recent studies reported that higher mesothelin expression was found to be associated with shorter patient survival; therefore, we examined the correlation of mesothelin overexpression with relapse-free survival (RFS) and overall survival (OS) in the EHBDCA patients. The group of 'high-level expression' of mesothelin had a significantly poorer RFS than the group of 'low-level expression' of mesothelin $(\mathrm{P}=0.026)$. In addition, the group of 'high-level expression' of mesothelin had a significantly poorer OS than the group of 'lowlevel expression' of mesothelin $(\mathrm{P}=0.022)$ (Fig. 3).

Luminal membrane expression of mesothelin is a prominent negative prognostic factor for the patients with EHBDCA. During our previous studies on pancreatic adenocarcinoma and gastric adenocarcinoma, we already noted that expression of mesothelin was found in the luminal membrane as well as in the cytoplasm (19). Mesothelin was reported to attach to the cell membrane through a glycosyl-phosphatidylinositol (GPI) anchor after being physiologically cleaved by some furin-like proteases (22), which are involved in the translocation of mesothelin, although the biological functions of mesothelin associated with its intracellular localization are not fully understood. Thus, we analyzed the intracellular localization of mesothelin by immunostaining to explore the clinicopathological significance of its translocation.

As shown in Table III, the group 'luminal membrane positive', which consisted of the cases with luminal membrane staining even partially, was $32(52.5 \%)$ cases, while the group 'luminal membrane negative', which contained 17 cases which were completely mesothelin negative was comprised of $29(47.5 \%)$ cases. The statistical analysis revealed that the incidence of luminal membrane positivity was significantly correlated with peritoneal metastasis $(\mathrm{P}=0.024)$ in addition to liver metastasis $(\mathrm{P}=0.006)$ (Table III). The analysis of the patients' overall survival showed that 'luminal membrane positive' of mesothelin indicated a significantly unfavorable RFS $(\mathrm{P}=0.012)$ and $\mathrm{OS}(\mathrm{P}=0.017)$ compared to 'luminal membrane negative' of mesothelin (Fig. 4).

To clarify the mesothelin expression as an independent prognostic factor, we performed a univariate analysis of the 61 EHBDCA using the Cox proportional hazards model, the result indicated that resection margin, 'high-level expression' and 'luminal membrane positive' of mesothelin were significantly correlated with risks of cancer mortality. Multivariate analysis also confirmed that resection margin (RR 3.361, 95\% CI, 1.670-6.763, $\mathrm{P}=0.0007)$ and 'luminal membrane positive' of mesothelin (RR 2.964, 95\% CI, 1.401-6.296, P=0.0045) were independent predictors of the overall patient survival (Table IV).

Isolation of 'cytoplasmic expression' of mesothelin potentiates more exquisite prediction of prognosis in EHBDCA. To explore the clinicopathological value of the cytoplasmic expression of mesothelin, we performed a sub-analysis in 'luminal membrane negative', dividing the group into 17 cases of 'mesothelin negative' and 12 cases of 'cytoplasmic expression'. The $\mathrm{P}$-value (OS, $\mathrm{P}=0.0085)$ between 'luminal membrane positive' and 'cytoplasmic expression' was minimum in these 


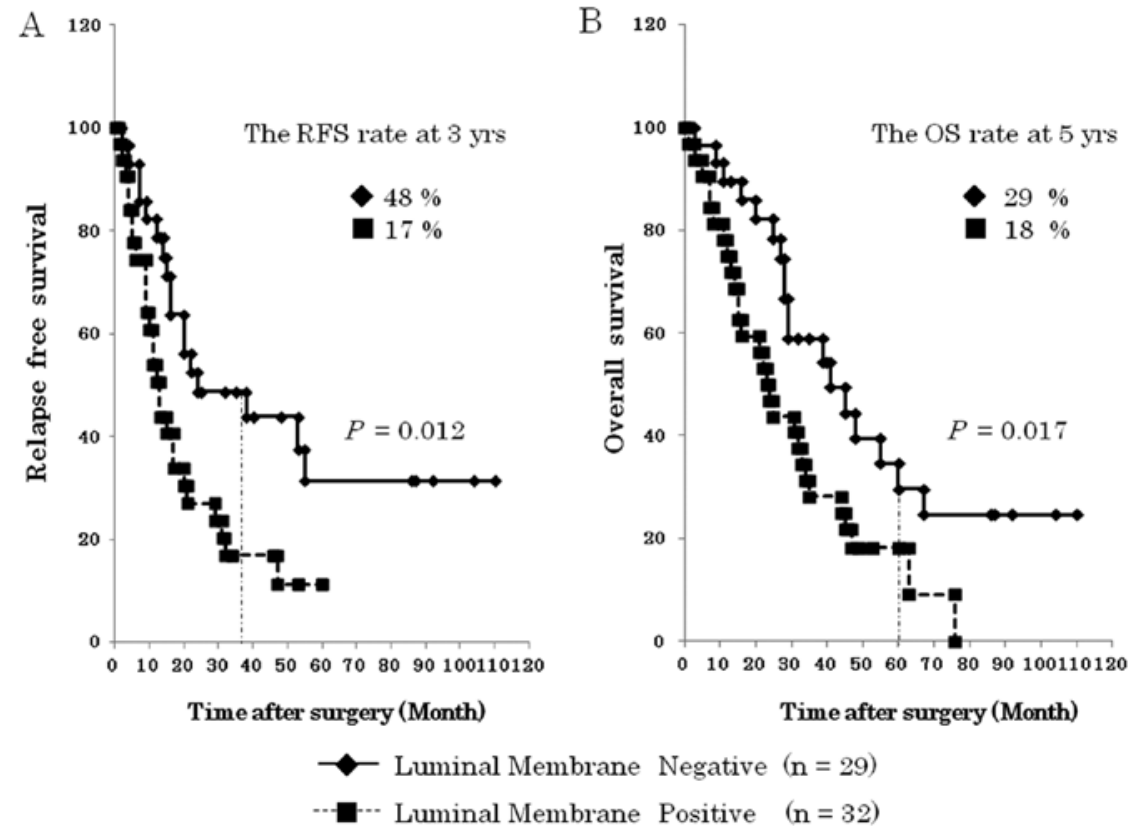

Figure 4. Relapse-free survival (RFS) and overall survival (OS) curves of EHBDCA patients according to the expression pattern of mesothelin. The group of 'luminal membrane positive' represented a statistically significantly unfavorable outcome compared to the group of 'luminal membrane negative' ( $\mathrm{P}=0.012$ and 0.017 , respectively).

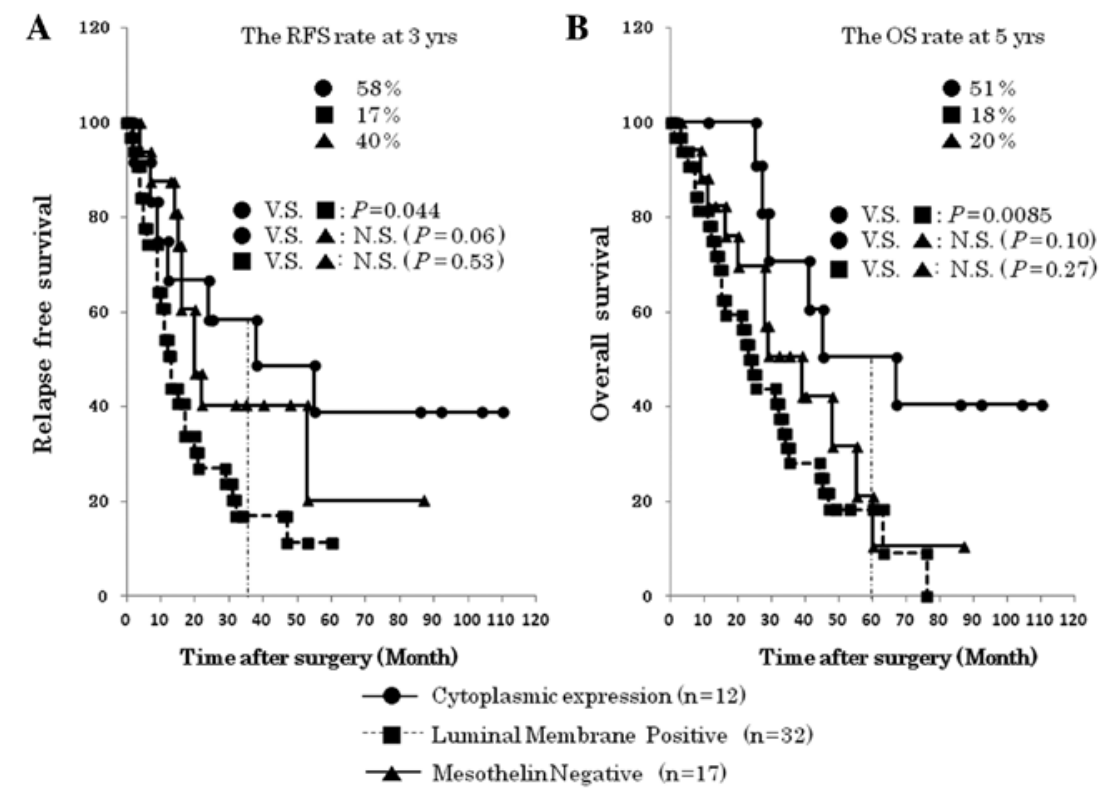

Figure 5. Relapse-free survival (RFS) and overall survival (OS) curves of EHBDCA patients among three groups of detailed expression patterns of mesothelin. 'Cytoplasmic expression' of mesothelin represented the best prognosis among the 3 groups.

survival analyses, suggesting the clinical benefit of isolation of 'cytoplasmic expression' of mesothelin (Fig. 5). Interestingly, 'cytoplasmic expression' of mesothelin represented relatively favorable patients' prognosis compared to 'mesothelin negative', although it was statistically not significant (RFS, $\mathrm{P}=0.06$; OS, $\mathrm{P}=0.10$ ).

\section{Discussion}

In this study, we confirmed that mesothelin expression is a prominent prognostic factor for EHBDCA patients as well as for other tumors such as pancreatic cancer and ovarian carcinoma described previously $(12,15,23)$. Furthermore, we revealed that the expression pattern of mesothelin, in luminal membrane or cytoplasm, could be a more evident prediction factor for these patients. These results evidently support our recent report of mesothelin expression patterns in gastric cancer in which luminal membrane expression, not cytoplasmic expression of mesothelin is a prominent negative prognostic factor for gastric cancer (19).

The mechanism for the membranous localization of mesothelin should be explained as follows: the full length of the 


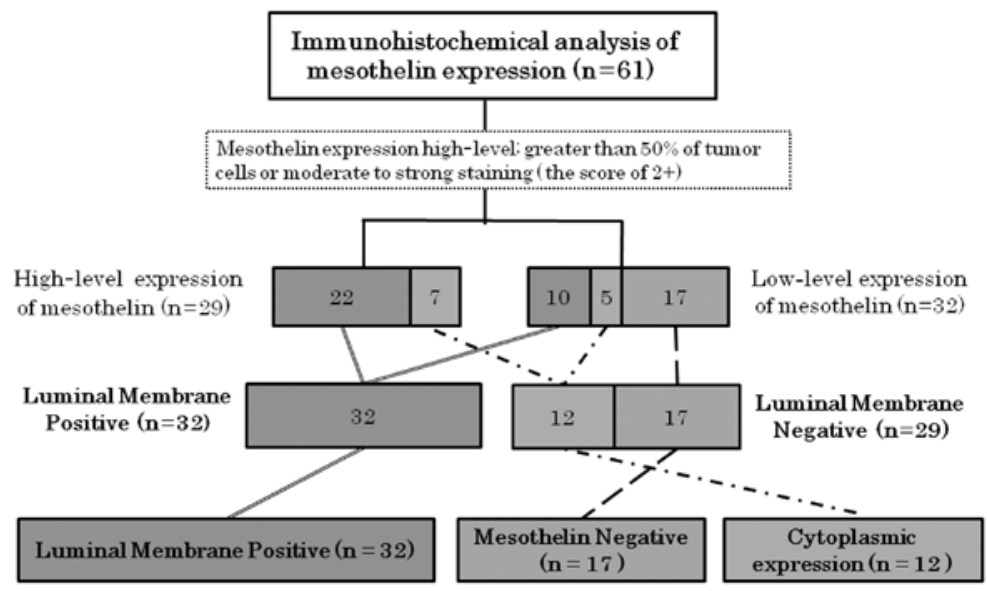

Poor

prognosis

$\underline{\text { Better }}$

Figure 6. Flow chart of immunohistochemical evaluation of mesothelin expression and the prognostic aspect. The $\mathrm{P}$-value (OS, $\mathrm{P}=0.0085)$ between 'luminal membrane positive' and 'cytoplasmic expression' was minimum in our survival analyses, suggesting the clinical benefit of isolation of 'cytoplasmic expression' of mesothelin.

Table IV. Univariate and multivariate analysis of patients' survival in EHBDCA.

\begin{tabular}{|c|c|c|c|c|c|c|}
\hline \multirow[b]{2}{*}{ Factor } & \multirow[b]{2}{*}{$\mathrm{n}=61$} & \multicolumn{2}{|c|}{ Univariate analysis } & \multicolumn{3}{|c|}{ Multivariate analysis } \\
\hline & & P-value & $\mathrm{RR}(95 \% \mathrm{CI})$ & $\mathrm{RR}(95 \% \mathrm{CI})$ & Hazard ratio & P-value \\
\hline \multicolumn{7}{|c|}{ Histopahological grade } \\
\hline 1 or 2 & 54 & 0.3931 & 1 & & $\mathrm{NC}$ & \\
\hline 3 & 7 & & $1.508(0.588-3.871)$ & & & \\
\hline \multicolumn{7}{|l|}{ pT-factor } \\
\hline pT1-2 & 32 & 0.4264 & 1 & & $\mathrm{NC}$ & \\
\hline pT3-4 & 29 & & $1.266(0.708-2.262)$ & & & \\
\hline \multicolumn{7}{|l|}{ pN-factor } \\
\hline Negative & 25 & 0.3639 & 1 & & $\mathrm{NC}$ & \\
\hline Positive & 36 & & $1.314(0.729-2.368)$ & & & \\
\hline \multicolumn{7}{|l|}{ pStage } \\
\hline I-IIB & 50 & 0.2026 & 1 & & $\mathrm{NC}$ & \\
\hline III-IV & 11 & & $1.608(0.774-3.339)$ & & & \\
\hline \multicolumn{7}{|c|}{ Lymphatic permeation } \\
\hline Negative & 23 & 0.1908 & 1 & & $\mathrm{NC}$ & \\
\hline Positive & 38 & & $1.537(0.807-2.924)$ & & & \\
\hline \multicolumn{7}{|c|}{ Blood vessel permeation } \\
\hline Negative & 26 & 0.2999 & 1 & & $\mathrm{NC}$ & \\
\hline Positive & 35 & & $1.370(0.756-2.482)$ & & & \\
\hline \multicolumn{7}{|c|}{ Perineural invasion } \\
\hline Negative & 9 & 0.4733 & 1 & & $\mathrm{NC}$ & \\
\hline Positive & 52 & & $0.728(0.306-1.732)$ & & & \\
\hline \multicolumn{7}{|c|}{ Resection margin } \\
\hline $\mathrm{pR} 0$ & 39 & 0.0398 & 1 & $1.670-6.763$ & 1 & 0.0007 \\
\hline $\mathrm{pR} 1$ & 22 & & $1.859(1.029-3.356)$ & & 3.361 & \\
\hline \multicolumn{7}{|c|}{ Mesothelin expression } \\
\hline Low-level & 32 & 0.0236 & 1 & $0.864-3.067$ & 1 & 0.1317 \\
\hline High-level & 29 & & $1.968(1.095-3.538)$ & & 1.621 & \\
\hline \multicolumn{7}{|c|}{$\begin{array}{l}\text { Luminal membrane expression } \\
\text { of mesothelin }\end{array}$} \\
\hline Negative & 29 & 0.0175 & 1 & $1.401-6.296$ & 1 & 0.0045 \\
\hline Positive & 32 & & $2.078(1.137-3.798)$ & & 2.964 & \\
\hline
\end{tabular}

RR indicates relative risk/hazard ratio; CI, confidence interval. NC, not calculable. 


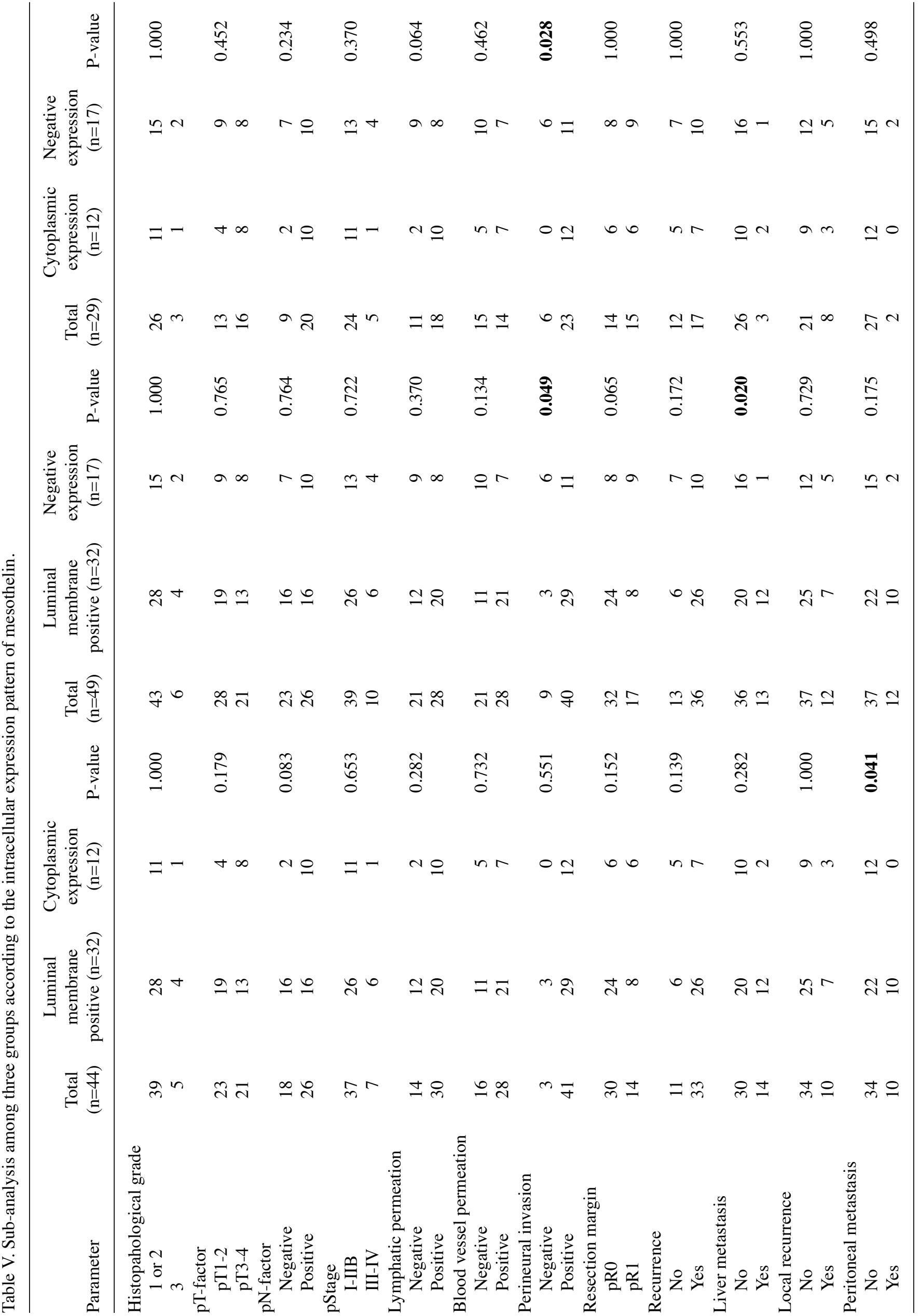


human mesothelin gene encodes a $71-\mathrm{kDa}$ precursor protein that is proteolytically cleaved by some furin-like proteases into an $N$-terminal secreted form and a $C$-terminal fragment, the 40-kDa mesothelin, which is a glycosyl-phosphatidylinositol (GPI)-linked glycoprotein $(6,13,15)$. Many researchers have investigated the role of the mesothelin expression in tumor biology and demonstrated the importance of mesothelin expression for tumor progression in vitro (14,24-26) and in vivo $(27,28)$; however, the clinicopathological significance of the membrane localization of mesothelin has not been clarified. The 5B2 anti-mesothelin antibody, which we employed here for IHC, can detect both the $71-\mathrm{kDa}$ precursor protein and the $40-\mathrm{kDa} C$-terminal fragment, but not the $30-\mathrm{kDa} N$-terminal fragment. According to the reported molecular processing mechanism of mesothelin and specificity of antibody, luminal membrane staining probably indicates the $40-\mathrm{kDa}$ membrane-bound form of mesothelin, while cytoplasmic staining would mean the $71-\mathrm{kDa}$ precursor form of mesothelin. Our results support the idea that the $40-\mathrm{kDa}$ membrane-bound form of mesothelin is an active form and promotes the aggressive features including increased cell motility, invasion or migration capabilities and growth of metastatic tumors $(24,25,29)$.

The fact that 'cytoplasmic expression' of mesothelin paradoxically resulted in better OS than mesothelin with 'mesothelin negative' took us by surprise (Fig. 5B). The RFS rate at 3 years (58 and 40\%, respectively) and OS at 5 years (51 and $20 \%$, respectively) were demonstrably better in 'cytoplasmic expression' compared to 'mesothelin negative', although the final RFS and OS were not statistically significant (RFS, $\mathrm{P}=0.06$; OS, $\mathrm{P}=0.10$ ). As indicated above, the majority of mesothelin in cytoplasm must be the $71-\mathrm{kDa}$ precursor form and might behave like a dominant negative form of mesothelin as a tumor suppressor. The conflicting results in some previous reports in which mesothelin expression was correlated with prolonged patient survival in gastric cancer (18) and in ovarian serous carcinoma (30), may be explained by confusing the luminal membrane and cytoplasmic expression of mesothelin. Isolation of 'mesothelin negative' might give us another disease entity, mesothelin-independent EHBDCA. The tumor cells in such a type of EHBDCA would obtain invasive ability without the association of mesothelin; therefore, this could indicate an alternative gene expression profiling. In fact, additional sub-analysis for clinicopathological parameters among the three groups showed interesting results. Frequent perineural invasion was observed in 'mesothelin negative' rather than in mesothelin positive cases even in luminal membrane or cytoplasm $(\mathrm{P}=0.049$ and 0.028 , respectively), while liver metastasis was abundantly found in 'luminal membrane positive' (Table V). Such conflicting results may suggest the distinct oncogenic process between mesothelin-associated and mesothelin-independent EHBDCA.

In terms of discovering the clinicopathological parameters, there are many previous studies demonstrating the prognostic significance of various molecules, such as epidermal growth factor receptor (EFGR) and c-erbB-2 (HER-2) in colorectal, breast and lung cancer (31). There are some other case reports describing a series of promising results targeting EGFR in patients with advanced biliary tract cancer (32-34); however, identification of useful prognostic markers for
EHBDCA still needs investigation. In addition, lack of effective adjuvant therapy against advanced EHBDCA requires establishing new therapeutic methods based on reliable molecular targeting markers; thus, mesothelin could be one of the potential targets for cancer molecular targeting therapy. Recombinant anti-mesothelin immunotoxin SS1P (CAT-5001) and a high affinity chimeric anti-mesothelin monoclonal antibody MORAb-009 recently entered phase II clinical trials $(35,36)$. To evaluate the therapeutic effect of such antibody-based medicine, pathological verification of membranous expression of the target molecule must be performed, because antibody-based drugs can usually access the molecules located on the cell membrane. We believe that luminal membrane expression of mesothelin in EHBDCA would be of clinical benefit not only as a prognostic factor but also as a predictive factor for the eligibility to mesothelintargeting therapies $(13,14,27,37,38)$.

In conclusion, we demonstrated the clinicopathological significance of the mesothelin expression as an independent prognostic factor. Moreover, identification of luminal membrane or cytoplasmic expression of mesothelin could be a reliable prognostic factor for EHBDCA and might offer a novel therapeutic strategy for patients with EHBDCA, including immunotherapy using peptide vaccine or monoclonal antibody therapy.

\section{Acknowledgements}

This research was supported by a Grant-in-Aid for Scientific Research (KAKENHI). The study sponsors had no involvement in the study design, in the collection, analysis and interpretation of data, in the writing of the manuscript, or in the decision to submit the manuscript for publication.

\section{References}

1. Ito K, Ito H, Allen PJ, et al: Adequate lymph node assessment for extrahepatic bile duct adenocarcinoma. Ann Surg 251: 675-681, 2010.

2. Ohashi M, Kusumi T, Sato F, et al: Expression of syndecan-1 and E-cadherin is inversely correlated with poor patient's prognosis and recurrent status of extrahepatic bile duct carcinoma. Biomed Res 30: 79-86, 2009.

3. Jarnagin WR, Fong Y, DeMatteo RP, et al: Staging, resectability, and outcome in 225 patients with hilar cholangiocarcinoma. Ann Surg 234: 507-519, 2001.

4. Akoad M and Jenkins R: Proximal biliary malignancy. Surg Clin North Am 88: 1409-1428, x-xi, 2008.

5. Veillette G and Castillo CF: Distal biliary malignancy. Surg Clin North Am 88: 1429-1447, xi, 2008.

6. Chang K and Pastan I: Molecular cloning of mesothelin, a differentiation antigen present on mesothelium, mesotheliomas, and ovarian cancers. Proc Natl Acad Sci USA 93: 136-140, 1996.

7. Chang K, Pastan I and Willingham MC: Isolation and characterization of a monoclonal antibody, $\mathrm{K} 1$, reactive with ovarian cancers and normal mesothelium. Int J Cancer 50: 373-381, 1992.

8. Argani P, Iacobuzio-Donahue C, Ryu B, et al: Mesothelin is overexpressed in the vast majority of ductal adenocarcinomas of the pancreas: identification of a new pancreatic cancer marker by serial analysis of gene expression (SAGE). Clin Cancer Res 7: 3862-3868, 2001

9. Hassan R, Kreitman RJ, Pastan I and Willingham MC: Localization of mesothelin in epithelial ovarian cancer. Appl Immunohistochem Mol Morphol 13: 243-247, 2005.

10. Ordonez NG: Value of mesothelin immunostaining in the diagnosis of mesothelioma. Mod Pathol 16: 192-197, 2003.

11. Ordonez NG: Application of mesothelin immunostaining in tumor diagnosis. Am J Surg Pathol 27: 1418-1428, 2003. 
12. Einama T, Kamachi H, Nishihara H, et al: Co-Expression of mesothelin and CA125 correlates with unfavorable patient outcome in pancreatic ductal adenocarcinoma. Pancreas 40 : $1276-1282,2011$

13. Hassan R, Bera T and Pastan I: Mesothelin: a new target for immunotherapy. Clin Cancer Res 10: 3937-3942, 2004.

14. Li M, Bharadwaj U, Zhang R, et al: Mesothelin is a malignant factor and therapeutic vaccine target for pancreatic cancer. Mol Cancer Ther 7: 286-296, 2008

15. Cheng WF, Huang CY, Chang MC, et al: High mesothelin correlates with chemoresistance and poor survival in epithelial ovarian carcinoma. Br J Cancer 100: 1144-1153, 2009.

16. Hassan R, Laszik ZG, Lerner M, Raffeld M, Postier R and Brackett D: Mesothelin is overexpressed in pancreaticobiliary adenocarcinomas but not in normal pancreas and chronic pancreatitis. Am J Clin Pathol 124: 838-845, 2005.

17. Swierczynski SL, Maitra A, Abraham SC, et al: Analysis of novel tumor markers in pancreatic and biliary carcinomas using tissue microarrays. Hum Pathol 35: 357-366, 2004.

18. Baba K, Ishigami S, Arigami T, et al: Mesothelin expression correlates with prolonged patient survival in gastric cancer. J Surg Oncol 105: 195-199, 2012.

19. Einama T, Homma S, Kamachi H, et al: Luminal membrane expression of mesothelin is a prominent poor prognostic factor for gastric cancer. Br J Cancer 107: 137-142, 2012.

20. Zhao H, Davydova L, Mandich D, Cartun RW and Ligato S: S100A4 protein and mesothelin expression in dysplasia and carcinoma of the extrahepatic bile duct. Am J Clin Pathol 127: 374-379, 2007

21. Sobin LH and Wittekind CW (eds): TNM Classification of Malignant Tumors. 6th edition. Wiley-Liss, New York, 2002.

22. Inami K, Kajino K, Abe M, et al: Secretion of N-ERC/mesothelin and expression of C-ERC/mesothelin in human pancreatic ductal carcinoma. Oncol Rep 20: 1375-1380, 2008.

23. Shimizu A, Hirono S, Tani M, et al: Coexpression of MUC16 and mesothelin is related to the invasion process in pancreatic ductal adenocarcinoma. Cancer Sci 103: 739-746, 2012.

24. Bharadwaj U, Marin-Muller C, Li M, Chen C and Yao Q Mesothelin overexpression promotes autocrine IL-6/sIL-6R trans-signaling to stimulate pancreatic cancer cell proliferation. Carcinogenesis 32: 1013-1024, 2011.

25. Bharadwaj U, Marin-Muller C, Li M, Chen C and Yao Q: Mesothelin confers pancreatic cancer cell resistance to TNF- $\alpha$ induced apoptosis through Akt/PI3K/NF- $\kappa \mathrm{B}$ activation and IL-6/Mcl-1 overexpression. Mol Cancer 10: 106, 2011
26. Chang MC, Chen CA, Hsieh CY, et al: Mesothelin inhibits paclitaxel-induced apoptosis through the PI3K pathway. Biochem J 424: 449-458, 2009

27. Hassan R, Schweizer C, Lu KF, et al: Inhibition of mesothelinCA-125 interaction in patients with mesothelioma by the anti-mesothelin monoclonal antibody MORAb-009: implications for cancer therapy. Lung Cancer 68: 455-459, 2010.

28. Bharadwaj U, Li M, Chen C and Yao Q: Mesothelin-induced pancreatic cancer cell proliferation involves alteration of cyclin $\mathrm{E}$ via activation of signal transducer and activator of transcription protein 3. Mol Cancer Res 6: 1755-1765, 2008.

29. Inami $\mathrm{K}$, Abe $\mathrm{M}$, Takeda $\mathrm{K}$, et al: Antitumor activity of anti-CERC/mesothelin monoclonal antibody in vivo. Cancer Sci 101: 969-974, 2009.

30. Yen MJ, Hsu CY, Mao TL, et al: Diffuse mesothelin expression correlates with prolonged patient survival in ovarian serous carcinoma. Clin Cancer Res 12: 827-831, 2006.

31. Hudis CA: Trastuzumab - mechanism of action and use in clinical practice. N Engl J Med 357: 39-51, 2007.

32. Huang TW, Wang CH and Hsieh CB: Effects of the anti-epidermal growth factor receptor antibody cetuximab on cholangiocarcinoma of the liver. Onkologie 30: 129-131, 2007.

33. Sprinzl MF, Schimanski CC, Moehler M, Schadmand-Fischer S, Galle PR and Kanzler S: Gemcitabine in combination with EGF-Receptor antibody (Cetuximab) as a treatment of cholangiocarcinoma: a case report. BMC Cancer 6: 190, 2006.

34. Bralet MP, Bellin MF, Guettier C, Adam R and Paule B: Response to cetuximab and gemcitabine-oxaliplatin in an advanced case of intrahepatic cholangiocarcinoma. Clin Oncol (R Coll Radiol) 18: $426,2006$.

35. Kreitman RJ, Hassan R, Fitzgerald DJ and Pastan I: Phase I trial of continuous infusion anti-mesothelin recombinant immunotoxin SS1P. Clin Cancer Res 15: 5274-5279, 2009.

36. Hassan R, Cohen SJ, Phillips M, et al: Phase I clinical trial of the Chimeric anti-mesothelin monoclonal antibody MORAb-009 in patients with mesothelin expressing cancers. Clin Cancer Res 16: 6132-6138, 2010.

37. Hassan R, Bullock S, Premkumar A, et al: Phase I study of SS1P, a recombinant anti-mesothelin immunotoxin given as a bolus I.V. infusion to patients with mesothelin-expressing mesothelioma, ovarian, and pancreatic cancers. Clin Cancer Res 13: 5144-5149, 2007.

38. Hassan R, Ebel W, Routhier EL, et al: Preclinical evaluation of MORAb-009, a chimeric antibody targeting tumor-associated mesothelin. Cancer Immun 7: 20, 2007. 